\title{
Information Preservation method application in low speed rarefied gas flow simulation
}

\author{
Bokang Yan ${ }^{1, a}$ \\ ${ }^{1}$ North China Electric Power University, Baoding 071000, China; \\ ayanbokang1994@outlook.com
}

Keywords: Micro scale, numerical simulation, DSMC method, IP method.

\begin{abstract}
Direct Simulation Monte Carlo (DSMC) is a widely used statistical numerical simulation method based on molecular motion. But in terms of the DSMC method. When we are simulating the gas flow at low speed, he molecular thermal motion speed is bigger than the macroscopic movement speed. If we want to get meaningful statistics, we need a great deal of sampling number. So the DSMC method is not suitable for simulation of gas flow at low speed. Jing Fan and Qing Shen come up with Information Preservation (IP) method. In this paper, on the basis of theoretical analysis, we improved Matlab codes for the DSMC calculation of Couette flow. We compared DSMC method with IP method in numerical simulation of micro scale one-component gas flow at low speed to validate feasibility and accuracy of IP in gas flaw at low speed.
\end{abstract}

\section{Introduction}

For micro scale flow and heat transfer, numerical simulation has many advantages. These advantages are especially important in the study of micro scale flow. It can make up for deficiencies in the experiment. DSMC method was first proposed by the Bird. In order to overcome the difficulties of DSMC. Which need to track a large number of particle motion. By tracking the movement of a limited number of molecular simulation to study the real gas flows. In this paper, the IP method is proposed based on the DSMC method. It overcomes the difficulties in the statistical simulation of low velocity of flow, which makes the information smaller than noise.

\section{Numerical simulation method}

\section{Introduction of IP method.}

IP method is a method embedded in the DSMC method. Mimicking molecules carry molecular thermal speed $c$ and information speed (IP speed) $u_{i}, c$ is used to calculate the molecular movement, collision and reflection on the ground. It fully complies with DSMC method. IP speed is used to record speed of per mimicking molecules, which represent huge number of real molecule. They don't affect the movement of molecules. IP speed sums macro velocity. When the molecules reflected from the surface, collide with each other or subjected to force action from the border into the information. IP speed updates.

\section{Program flow of IP method}

The main steps:

1. Distribute the heat velocity $c_{k}{ }^{\prime}$ and IP speed $c_{0}$ of Mimicking molecules in flow field

2. Set IP speed $c_{k}$ according to the flow speed of the initial conditions.

3. Moving each molecule at the speed of molecular heat.

4. Changing $c_{k}$ in $\Delta t$ according to the following conditions:

a. When a collision occurs with a solid wall, the velocity of IP $c_{k}$ is consistent with the velocity of the molecule reflected from the wall.

b. Setting the velocity of simulated particle from the boundary to the flow field according to boundary conditions.

c. When considering the external force field, Taking the grid as the basic unit to calculate the 
acceleration of simulated molecules.

$$
a=\frac{F}{\rho \Delta V}
$$

$\mathrm{F}$ is the total force of external force on the grid; $\rho$ is gas density; $\Delta V$ is volume of gas in a grid; acceleration $a$ increases the speed of molecules in grids with a increment $\Delta u=a \Delta t$ in $\Delta t$.

$\mathrm{d}$. When two simulation particles collide, the velocity variation of simulated molecules is divided into two parts. The velocity of thermal motion is consistent with the DSMC method, IP speed using the following formula:

$$
c_{1}{ }^{\prime}=c_{2}{ }^{\prime}=\frac{m_{1} c_{1}^{*}+m_{2} c_{2}^{*}}{m_{1}+m_{2}}
$$

Superscript “*” and “, ” represents the speed before the collision and after collision.

5. Calculate the speed of the fluid velocity macro According to IP speed. using the following formula:

$$
U=\frac{1}{N} \sum_{K=1}^{N} c_{k}
$$

If the flow is steady, repeat steps 3 and 4 until the results reached a steady state. Then, repeat steps 3 to 5 , sampling simulation of molecular in order to get macro-flow information. If the flow is not steady, Repeat steps 2 to 5

\section{The programs verification of DSMC method and IP method and Numerical simulation analysis of Couette flow}

In this paper, we verified the DSMC method and IP method. Then we compered the result. We choice the gas in calculation is argon. The speed of the wall $V_{W}$ changed from $3 \mathrm{~m} / \mathrm{s}$ to $300 \mathrm{~m} / \mathrm{s}$. The characteristic scale of Couette flow is $\mathrm{L}=1 \mathrm{~m}$. $\mathrm{Kn}=0.063$. Total number of simulated molecules is 1250. Total number of cycles is 10000. Following the results of the calculations are discussed in detail. Speed comparison diagram as shown in the following figures. We simulated the wall speed changing Red line is the velocity distribution of the gas in the figure, left figures is the result of DSMC method, the figures on the right are results of IP method.

$V_{W}=300 \mathrm{~m} / \mathrm{s}$

DSMC method:

IP method:

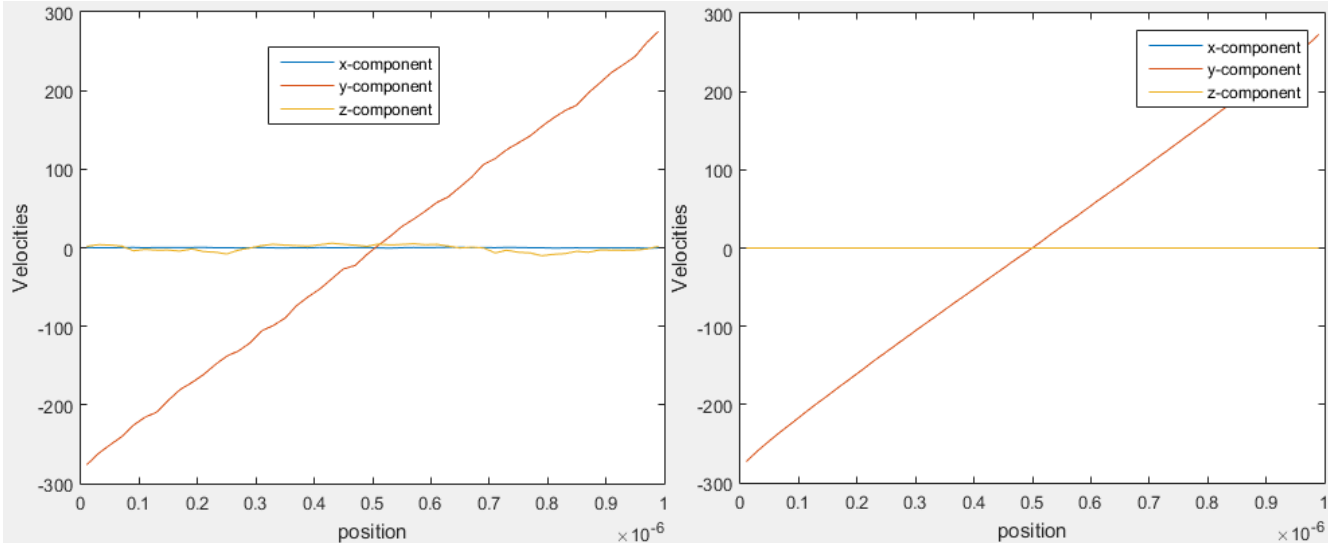

$V_{W}=30 \mathrm{~m} / \mathrm{s}$

DSMC method:

IP method: 

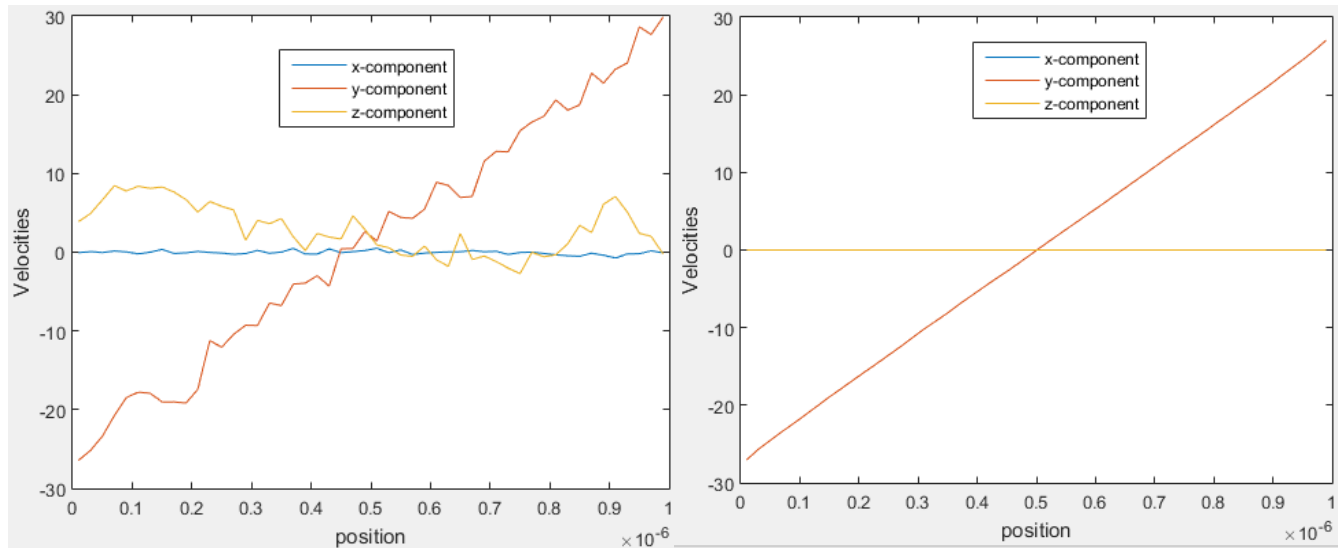

$V_{W}=3 \mathrm{~m} / \mathrm{s}$

DSMC method:

IP method:
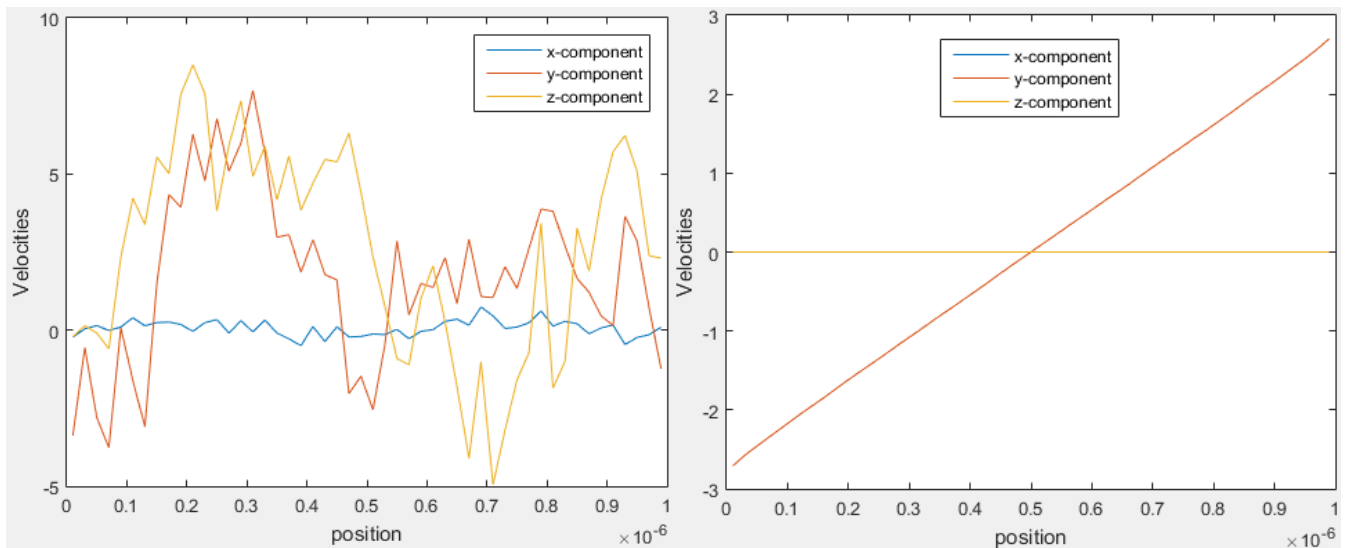

As can be seen from the horizontal contrast diagram, when the speed of the wall is larger the result of DSMC method is close to the result of IP method. When the speed of the wall is small, the result of DSMC method become disordered, which is inconsistent with the actual results. But the result of IP method is still a straight line, which is close to the actual results. We can draw a conclusion. IP method has high precision in low wall speed. The precision of DSMC method is low.

\section{Summary}

IP method is a good way in one-dimensional simulation of rarefied gas flow between two flat

The result of IP method is close to DSMC method and the experimental data. Especially when simulating low speed rarefied gas flows, DSMC method has very large deviation, IP method could still be better accuracy. It has greater advantages.

\section{References}

[1]Qing Shen. Rarefied gas dynamics[M]Beijing: Defense industry publishing house,2003

[2] Mingqiao Wu. Study on hybrid algorithm for DSMC/EPSM of rarefied gas flow [D] National University of Defense technology, 2001.DOI:10.7666/d.y481926.

[3] M. N. Macrossan. Matlab codes for the DSMC calculation of Couette flow,

using the variable-hard-sphere (VHS) collision model. Department of Mechanical Engineering Report No. 2009/02 